\title{
Research Paper \\ Developing a structural model of the quality of the main family and the tendency to infidelity between spouses through the mediation of self-differentiation
}

\begin{abstract}
Citation: Shaghaghi F, Aghayosefi A, Mirzahoseini H. Developing a structural model of the quality of the main family and the tendency to infidelity between spouses through the mediation of selfdifferentiation. J of Psychological Science. 2022; 20(108): 2327-2341.
\end{abstract}

Fatemeh Shaghaghi $^{1}$, Alireza Aghayosefi ${ }^{2}$, Hasan Mirzahoseini ${ }^{3}$

1. Ph.D Student in General Psychology, Qom Branch, Islamic Azad University, Qom, Iran.

2. Associate Professor, Department of Psychology, Payame Noor University, Tehran, Iran.

3. Assistant Professor, Department of Psychology, Qom Branch, Islamic Azad University, Qom, Iran.

URL: https://psychologicalscience.ir/article-1-1195-fa.html
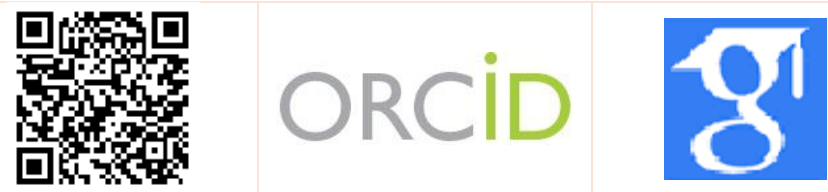

$\underline{10.52547 / J P S .20 .108 .2327}$

\section{A R T I C L E I N F O A B S T RA C T}

Keywords:

Self - differentiation, Quality of the main family,

Tendency to infidelity

Received: 26 Mar 2021 Accepted: 01 May 2021 Available: 20 Feb 2022
Background: Many studies emphasize the impact of child-parenting relationships and family interactions on future compromises, including commitment after marriage. Meanwhile, the development of the concept of self-differentiation within the family, which means emotional regulation and anxiety management, can affect the relationship between spouses. However, the relationship between these variables in a structural model has not been studied in research.

Aims: The purpose of this study was to develop a structural model of the quality of the main family and the tendency to infidelity the through the mediation of self-differentiation.

Methods: This was a correlational study and the statistical population of the study included all married people in Tehran city at 1999 year. The sample consisted of 349 people who were selected by random sampling method. Research instruments included the Stout et al.'s Family Quality Scale (1985), the Scorne and Friedlander Self-Differentiation Scale (1989), and the Mark Watley (2006) Infidelity Scale, which were completed by participants. Also, in order to investigate the relationships between variables, the method of structural equations, correlation test between variables and bootstrap test were used.

Results: Evaluation of the hypothetical model of the research showed that the hypothetical model fits with the measurement model $(\mathrm{CFI}=0.98$, IFI $=0.98$, RMSEA $=0.056)$. Also, the quality of the main family is not directly related to the tendency to breach of contract (0.03) and indirectly affects tendency to infidelity through self-differentiation (0.20). In other words, self-differentiation plays a mediating role in the relationship between the quality of the main family and tendency to infidelity $(-0.24)$.

Conclusion: Poor family quality, which leads to low levels of differentiation in individuals, is likely to lead to tendency to infidelity in the next life with the spouse. More research is needed to improve the quality of parenting relationships.

* Corresponding Author: Alireza Aghayosefi, Associate Professor, Department of Psychology, Payame Noor University, Tehran, Iran.

E-mail: arayeh1100@gmail.com

Tel: (+98) 9121519023

2476-5740/ (C) 2021 The Authors. This is an open access article under the CC BY-NC-ND license

(https://creativecommons.org/licenses/by-nc/4.0/). 


\section{Extended Abstract}

\section{Introduction}

Studies show that the quality of the main family plays a very important role in the future life of children; According to Weiss (2014) the quality of main family has a lasting effect on the success and happiness of future married life. Studies also show the connection between a previous experiences in the main family and his subsequent romantic relationships (Tughrilipour et al., 2018). The quality of the main family predicts characteristics such as independence, intimacy, compromise, satisfaction and quality of communication between people. Therefore people whose family quality is positive, the quality of their subsequent interactions is more desirable, and they are more likely to succeed in their future lives. Considering this, Bowen's multi-generational theory states that individuals learn the foundation of interpersonal connections in their main family and couples' conflicts are a continuation of the problems of their main family connection. The problems of the main family, such as interpersonal conflicts are comforted in extramarital relationships and affect their beliefs, attitudes, behaviors, self-esteem and interactive patterns (weiss, 2014).

One of the destructive effects of the negative performance of the main family that occurs in subsequent spousal connections is infidelity. By definition, any sexual or emotional connection goes beyond the framework of a committed connection between two spouses is called infidelity or breach of contract According to studies, the number of people who considered the breach of contract to be incorrect decreased between 2000 and 2016, and the number of people who considered this type of connection to be correct increased (Blau and Hartnett, 2005).

A review of the research background in the field of infidelity shows that most previous studies in this field have focused on two main areas: The first area of research focused on the definitions and types of relationships, identifying related variables such as gender differences, age, education, religious beliefs, cultural differences, duration of Marriage, level of sexual satisfaction, etc. Researchers in this field have considered and studied the connection of different variables with infidelity (Glass and Wright,1992; Atkins, et al., 2001; Blastin and Swartz, 1983; Teres and Gaissen, 2000). The second area of research has studied the application and effectiveness of various therapies in the treatment of injuries caused by infidelity in the relationship between spouses (Imanizad et al.,2021). Recent research addresses the question of whether the management of anxiety and emotion is a factor in the occurrence of infidelity (Hertlin and Skiggs, 2005; Hertlin, Ray, Wechler and Kilmer, 2003; Fish et al., 2012; Behnam, 2015).

Self-differentiation is the concept that Bowen used to describe the way people manage their anxiety in a relationships. Self-differentiation is defined as the ability to regulate one's emotions and the balance between individuality and integration (Priest, 2019). The marital system of differentiated couples adapts well when faced with conflict and shows less anxiety and emotional reactions. On the other hand, couples with low differentiation in the face of conflict experience high anxiety and avoid the situation and become more involved in emotional defense mechanisms such as emotional cutoff, fusion or triangulation (Lampis et al., 2019). Bowen believed that the degree of differentiation that individuals achieve in their main family has a significant and lasting impact on their lives (Qarabagh, 2011). Selfdifferentiation can be considered as a process and result of family functioning, and this is one of the most vital personality traits for maturity and mental health (Lampis et al., 2019).

According to previous research, since there are few studies on the connection between variables and has not been studied in the structural model, thus this study intends to examine the direct And indirectly effects the variables in the structural model. So the question is: does the main family quality connection with the tendency to infidelity, and does the selfdifferentiation mediate the connection between the main family quality and the tendency to infidelity? 


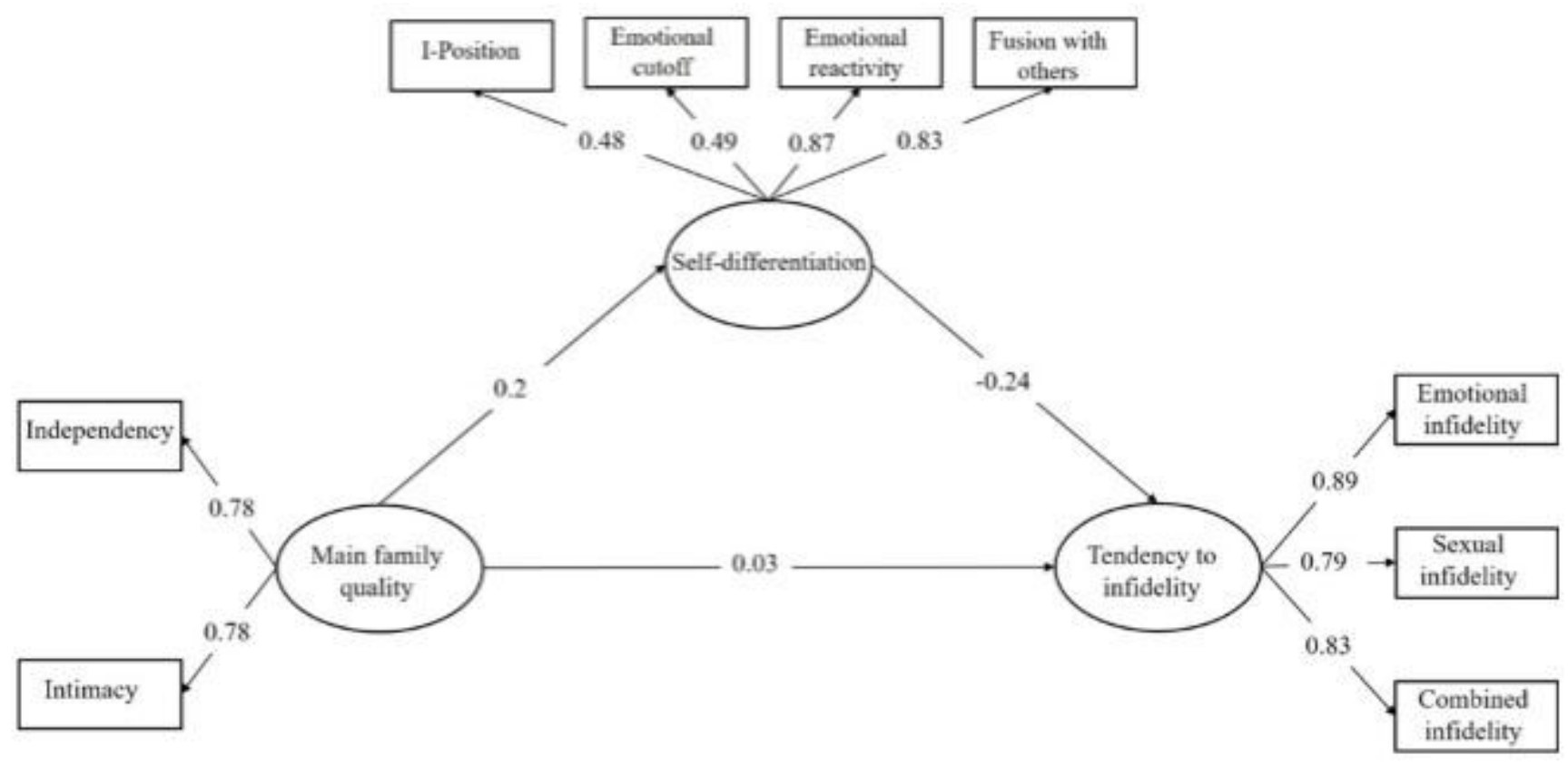

Figure 1. Structural Model of Research

\section{Method}

The present study is one of the basic researches and the method of data collection is correlation and structural equation modeling. The target population was all married people in Tehran city at 1999 year. The research sample was consisted of 349 married people. They after obtaining consent and stating the confidentiality of information, ensuring no harm (financial, physical and psychological) and the autonomy of the company by random sampling method were selected. Research instruments included the Stout et al.'s Family Quality Scale (1985), the Scorne and Friedlander Self-Differentiation Scale (1989), and the Mark Watley (2006) Infidelity Scale, which were completed by participants. Inclusion criteria were at least 20 years old and married. Because in this study we were looking to investigate the complex connections between variables, structural equation modeling (using LISREL 8.80 software), correlation test between variables and bootstrap test were used. The structural equation model method tests the pattern in two steps, which include the measurement pattern test and the structural test. The measurement model evaluates the validity and reliability of measurement tools and research structures. The structural model measures the connections of latent variables.

\section{Results}

Considering the Structural equation modeling is a statistical method based on matrix-covariance, so before evaluating the measurement model and structural model, the important assumptions for structural equation modeling include the normality of univariate and multivariate and the absence of multiple alignments was examined. In order to evaluate the normality of univariate, skewness and elongation of visible variables were performed. The skewness of the observable variables was in the range of -0.18 to 2.1 and their elongation was in the range of 3.9 to -0.20 . According to Kitner and Miller (1990), the cut-off point of $3 \%$ is appropriate for the amount of skewness. In this study, the assumption of multivariate normality was investigated by calculating the relative multivariate elongation index, the value of which was 1.08. If the value of this index is not more than 3 , the normality of multivariate is realized. Therefore, the distribution of all combinations of variables is normal. 
Table 1. Indicators of fit of measurement model and structural model of research

\begin{tabular}{|c|c|c|c|c|c|c|c|c|c|}
\hline Variables & $X^{2}$ & df & $\chi^{2} / \mathrm{df}$ & RMSEA & SRMR & CFI & IFI & GFI & AGFI \\
\hline Measurement model & 46.55 & 23 & 2.02 & 0.054 & 0.067 & 0.98 & 0.98 & 0.97 & 0.94 \\
\hline Structural model & 46.55 & 23 & 2.02 & 0.054 & 0.067 & 0.98 & 0.98 & 0.97 & 0.94 \\
\hline Independent variable & Mediator var & & Depend & variable & $\begin{array}{l}\text { Upper } \\
\text { limit }\end{array}$ & $\begin{array}{c}\text { Lower } \\
\text { limit }\end{array}$ & \multicolumn{2}{|c|}{$\begin{array}{l}\text { Estimation } \\
\text { error }\end{array}$} & $\begin{array}{c}\text { Effect } \\
\text { size }\end{array}$ \\
\hline $\begin{array}{l}\text { The quality of the } \\
\text { main family }\end{array}$ & Differentiat & & $\begin{array}{r}\text { Ten } \\
\text { in }\end{array}$ & $\begin{array}{l}\text { y to } \\
\text { ity }\end{array}$ & -0.010 & -0.088 & \multicolumn{2}{|c|}{0.020} & 0.04 \\
\hline
\end{tabular}

The default absence of multiple alignment was checked by examining the correlation matrix between the visible variables. Examination of this matrix indicates that there is no multiple alignment between them. Correlation coefficients are in the range of $0.00001<\mathrm{r}<791$. Correlation coefficients above 0.85 make it difficult to accurately estimate the model. In such cases, one of the two variables should be excluded from the analysis. The maximum likelihood estimation method was used to evaluate the measurement model and the structural model. The measurement model identifies the connection between visible variables and latent variables. Evaluation of this model was performed using confirmatory factor analysis. The fit indices of the measurement model, presented in Table 1, show the optimal fit of this model. Thus, visible variables have the ability to operate latent variables.

Figure 1 illustrates a conceptual structural model with standard coefficients. According to figure the main family with standard coefficient of 0.03 (t-values $=$ 0.49 ), 0.2 (t-values $=3.22$ ) affect the tendency to infidelity and self-differentiation. Also selfdifferentiation with a standard coefficient of -0.24 (tvalues $=-78.78)$ affects the infidelity. In the structural model, the significance of the path coefficient is determined using the value of $\mathrm{T}$. If the value of $\mathrm{T}$ is more than 1.96, the connection between the two structures is significant, so all paths except the direct path of main family to infidelity is significant.

In the present study, Bootstrap test was used to evaluate the mediating connections. Bootstrap provides the most powerful and logical way to evaluate indirect effects. The significance evaluation of these connections can be examined in two ways: the first method by referring to the levels of significance and the second method by examining the confidence intervals. If the upper and lower limits with $95 \%$ confidence interval are also marked for the mediating path (both positive or both negative) or in other words, the value of zero is not placed between these two limits, the desired path is significant at $\mathrm{p}<$ 0.05 (Table 2).

\section{Conclusion}

The findings indicate that quality of the main family does not directly affect the tendency to infidelity. The results of this study also showed that quality of the main family and tendency to infidelity are related to mediation of self-differentiation. The results of this study are based on the findings of Schwartz and Tigpan (2006), Lampis (2019), Naimi et al. (2016), Calta (2014), Skorn and Stanley (2009), Hertlin et al. (2003), Fish et al. (2012) and Behnam (2015) are conformity.

The first time Multrap stated that infidelity is an emotional solution to an emotional problem. In fact, person with poor self-differentiation reduces their anxiety level by triangulation (Hertlin et al., 2003). Likani (2013) in research on the connection between self-differentiation and violence in marriage states that high self-differentiation prevent spouses from merging in critical situations and enable them solve problems not based on emotional responsiveness. On the other hand, undifferentiated couples use emotional reactivity and move towards triangulation and infidelity in difficult situations. These results are consistent with other studies that show that selfdifferentiation has a direct negative effect on the tendency to infidelity (Zarei et al., 2014; Glad, 1999). In explaining the mediating role of selfdifferentiation in the connection between the main family and infidelity, It can be said that the family as the first base of personality formation is the most 
basic factor in the process of differentiation. The families in good health have a high rate of "self" growth in their members, thus the individual's differentiation increases and act more independently. Differentiated people have a clear definition of themselves and their beliefs. They can choose their direction in life and not lose control in highly emotional situations with reason and logic in mind. In contrast, undifferentiated individuals who do not have a defined identity and move on the emotional wave of the family, experience high levels of chronic anxiety and are prone to psychological problems. They try to maintain a dynamic balance in their connection by communicating with someone other than their spouse. Thus, people raised in troubled families are more likely to repeat past experiences and observations in their future lives. Although no study has been found on the connection between main family and infidelity, the role of the family in the formation or persistence of mental disorders is known well and a large amount of research findings on this subject(Fish et al., 2012).

\section{Ethical Considerations}

Compliance with ethical guidelines: This article is taken from a PhD thesis of the first author in the field of general psychology in Qom Islamic Azad University, which has an ethics code with the number IR.IAU.QOM.REC.1399.058, and the participants participated in the research consciously and voluntarily.

Funding: This study was conducted as a $\mathrm{PhD}$ thesis with no financial support.

Authors' contribution: The first author is the senior author, the second and third authors are the supervisor and the advisor, respectively.

Conflict of interest: The authors declare no conflict of interest for this study.

Acknowledgments: I would like to thank the supervisors and advisors as well as the people who participated in this research. 


\section{تدوين الكوى ساختارى كيفيت خانواده اصلى و Fرايش به بيمانشكنى بين همسران با ميانجى كرى تمايزيافتكى خود}

\section{فاطمه شقاقى'، عليرضا آقايوسفى "، حسن ميرزاحسينى'}

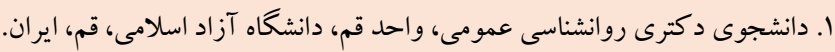

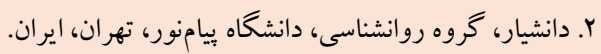

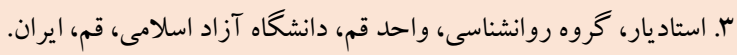

\section{جكيده}

زمينه: يثزوهشهاى زيادى به تأثير رابطه والدگرى - فرزندى و تعاملات خانوادكى بر سازش جويىهاى آينده از جمله تعهد بعد از ازدواج

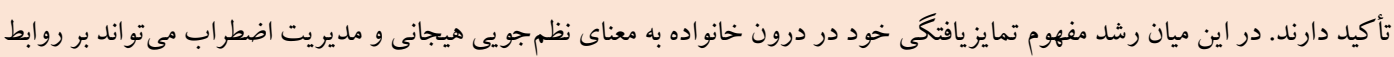

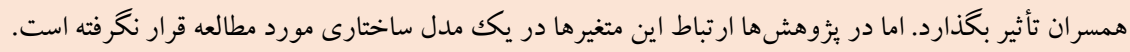
هدف: هدف از اين يزوهش تدوين الكوى ساختارى كيفيت خانو اده اصلى و گرايش به بيمانشكنى با ميانجى گرى تمايز يافتخى خود بود.

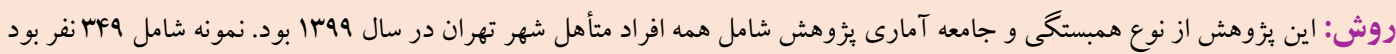

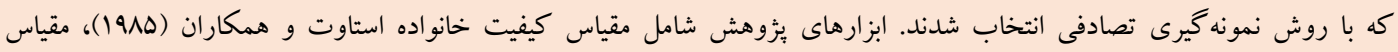

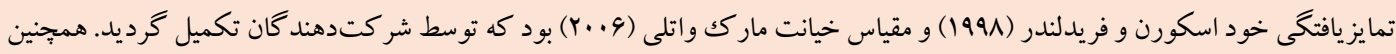

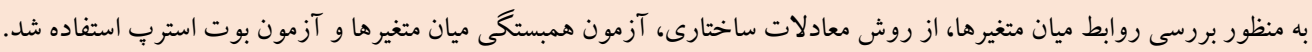

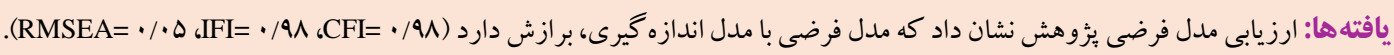

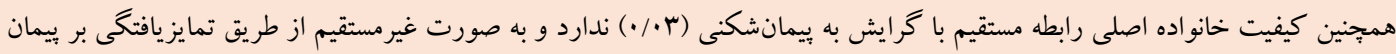

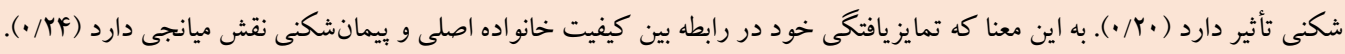

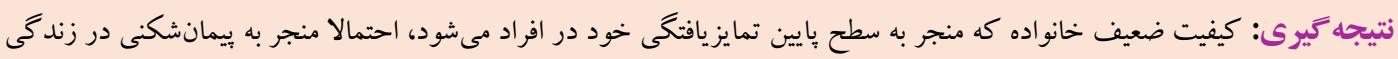

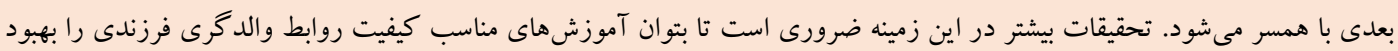

مشخصات مقاله

كليدوازمها: تمايزيافتكى خود، كيفيت خانو اده اصلى، كرايش به بيمانشكنى 
ياد مى گيرند و مشكلات خانوادگى و تعارضات زوجها ادامه مشكلات رابطه خانواده اصلى آنها است. مشكلات خانو اده اصلى مانند تعارضات

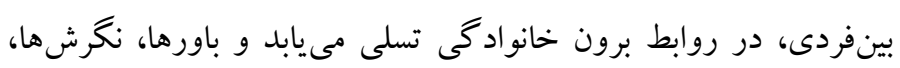

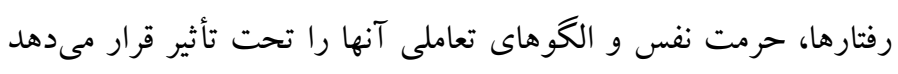

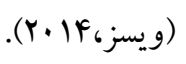

بر اساس مطالعات، شمار افرادى كه بيمانشكنى را نادرست مىدانستند،

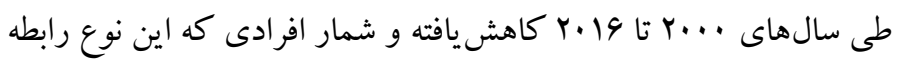

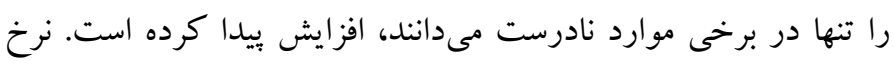

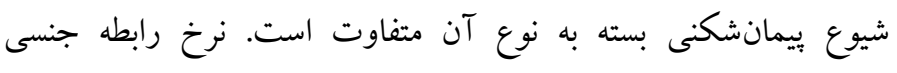

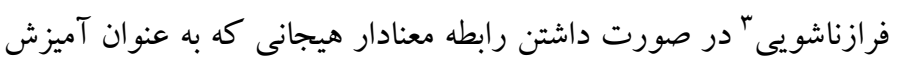

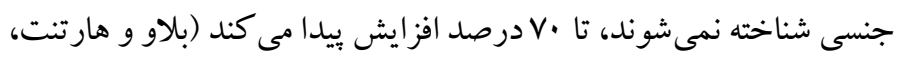

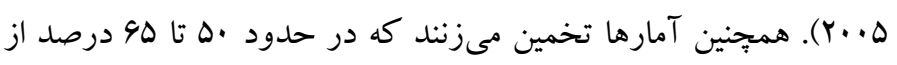

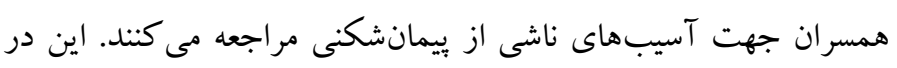
صورتى است كه از نظر زوج درمانگران بيمانشكنى از حادترين و مشكل

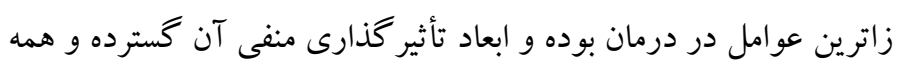

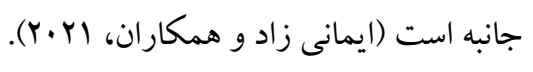

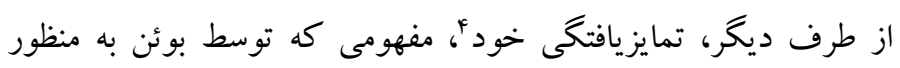
توصيف شيوهاى كه فرد اضطراب خود را در رابطه مديريت مى كند، بكار كرفته شد و اشاره دارد به اينكه فرد از طريق شيوهاى الكويافته (به عنوان

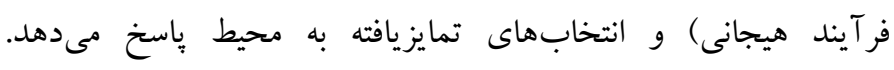

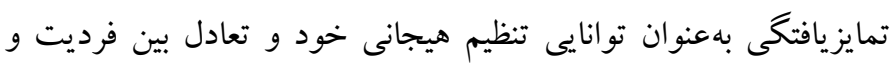

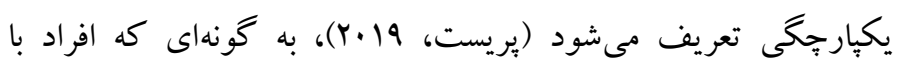
تمايزيافتكى بالا واكنش هاى خود را به صورت منطقى كنترل منى مئى كنند و

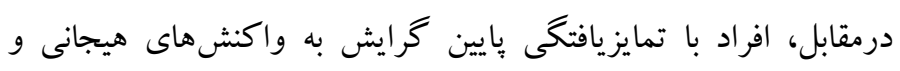

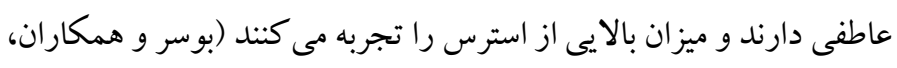

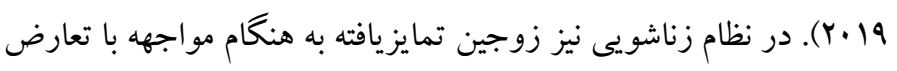

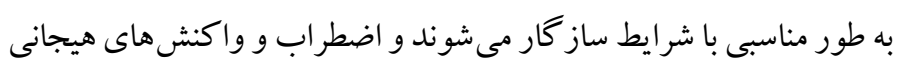
كمترى از خود نشان مىدهند. در سمت مقابل، زوجين با تمايزيافتخى بايين

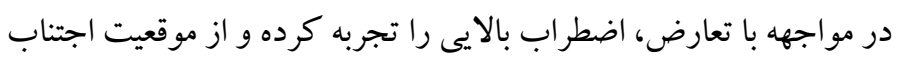

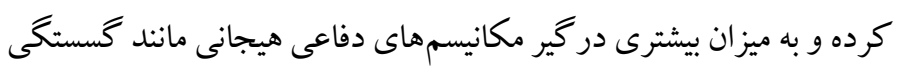

3. extramarital

${ }^{4}$. self- differentiation
مقدمه

بررسىها نشان مىدهند كه كيفيت خانواده اصلى' در زندگى آينده

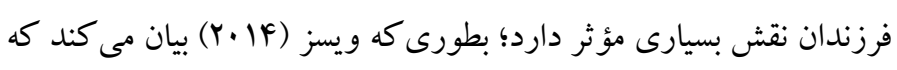

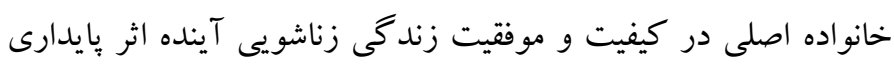

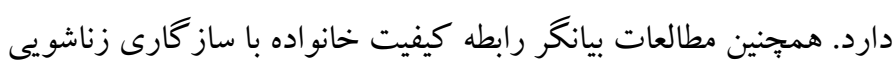

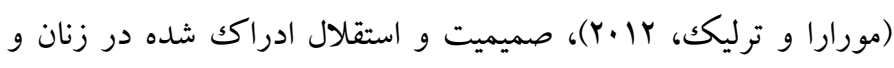

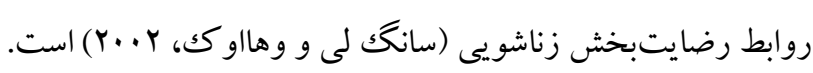

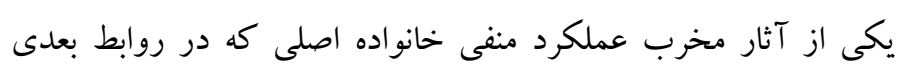

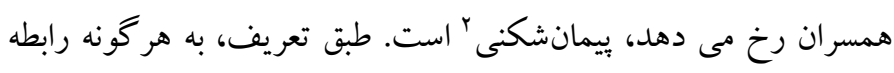

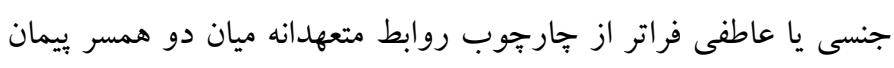

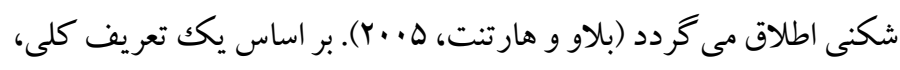

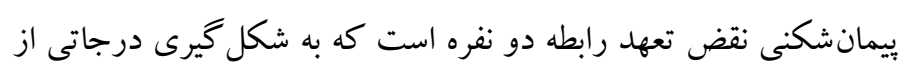
صميميت عاطفى و فيزيكى با فردى خارج از اين رابطه منجر مى نشود.

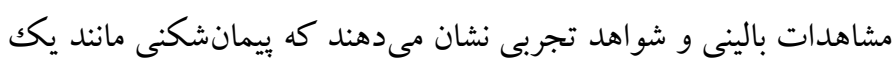

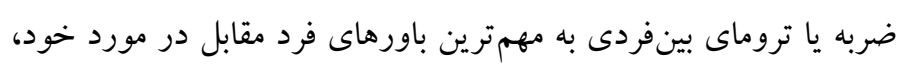

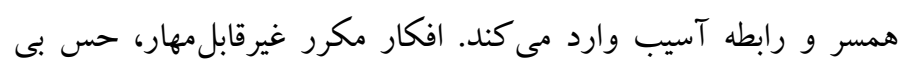

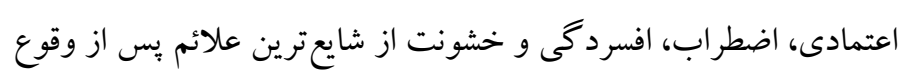

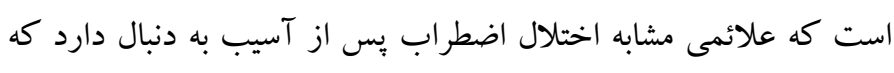

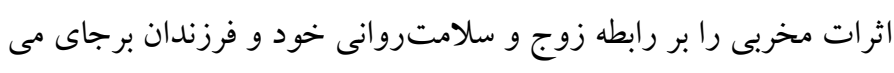

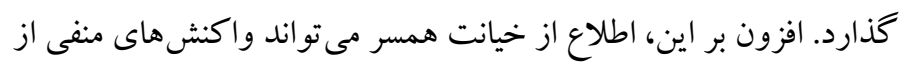

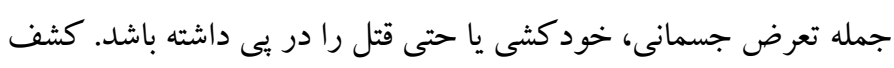
خيانت و بحران ناشى از آن در صورتى كه خاتمه نيابد تأثيرات منفى بلنى دئد

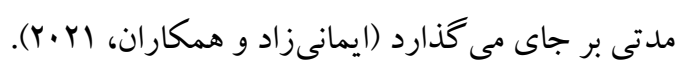

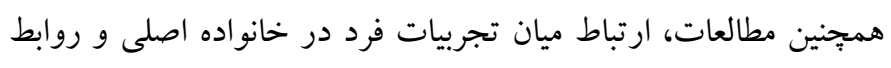

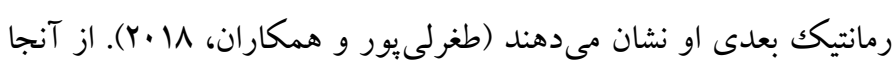

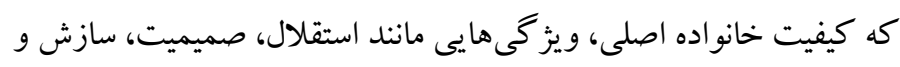

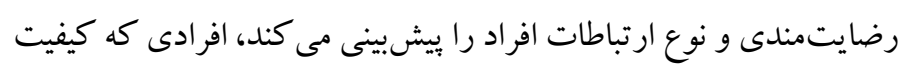

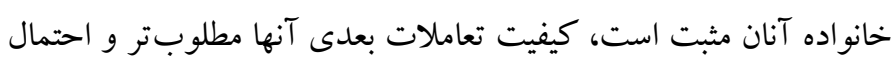

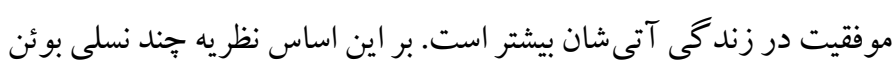

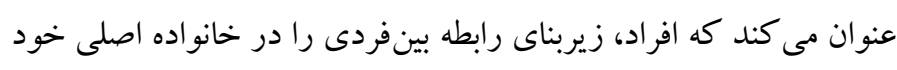

1. quality of the main family

2 . infidelity 
با بيمانشكنى مدنظر قرار دادهاند. حوزه دوم بثزوهشهاى انجام شده،

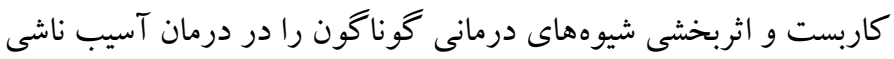

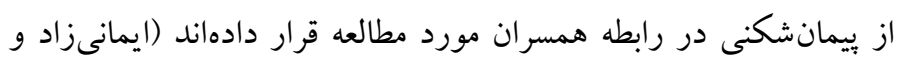

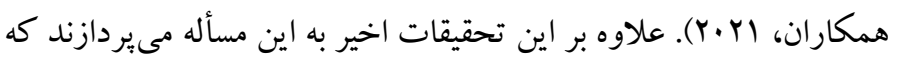

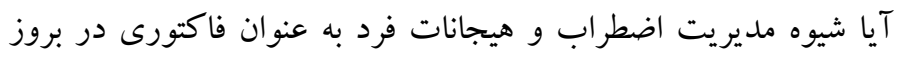

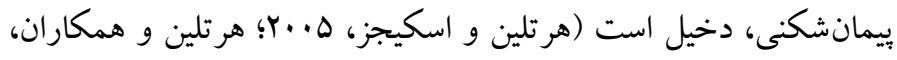

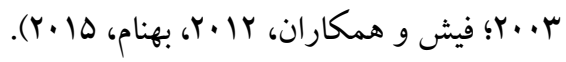
با توجه به تحقيقات بيشين، از آنجا كه مطالعات اندككشمارى در خصوص

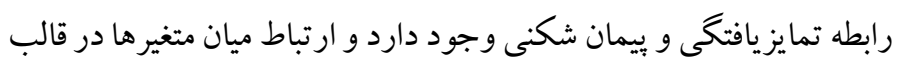

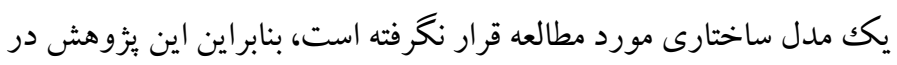

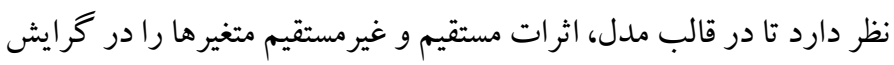
به بيمانشكنى مورد بررسى قرار دهد. لذا سؤال اين است: آيا كيفيت

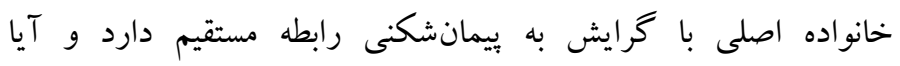

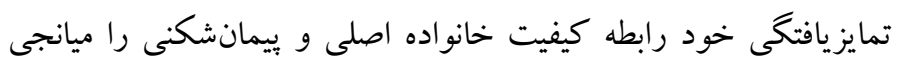
كرى مى كند؟ الف) طرح ئوهش و شركت كنند كان: يزوهش حاضر از نظر هدف

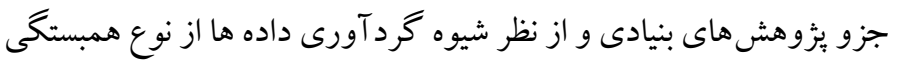
و مدلسازى معادلات ساختارى است. جامعه هدف، كليه افراد متأهل شهر

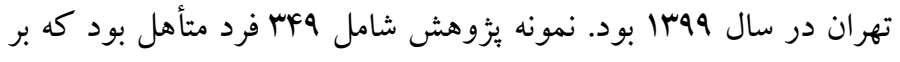

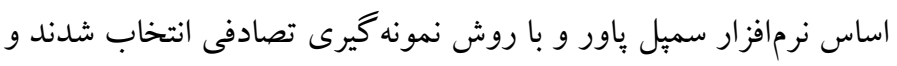

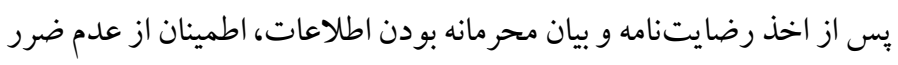

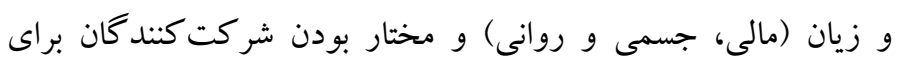

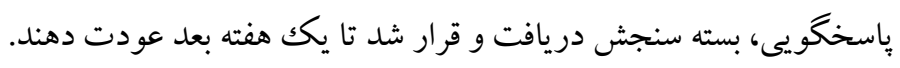
با توجه به موقعيت ناشى از شرايط بيمارى كرونا بستهاى سنجش يس از

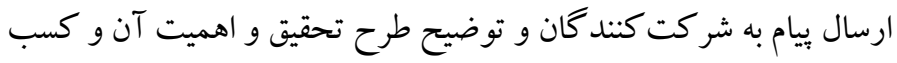
رضايت آكاهانه، به صورت الكترونيكى براى آنان ارسال گرديد. از ميان

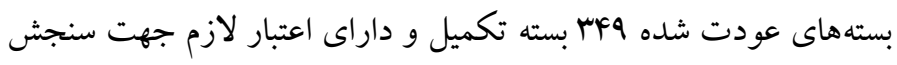

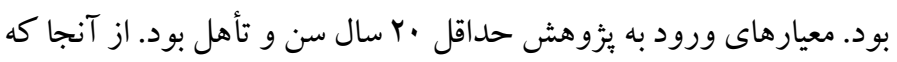
در اين يزوهش به دنبال بررسى روابط بيجيجيده ميان متغيرها بوديم، از مدل
عاطفى، همجوشى يا مثلثسازى مىشوند (لمييس و همكاران، 19. (Y).

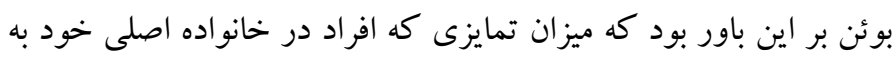

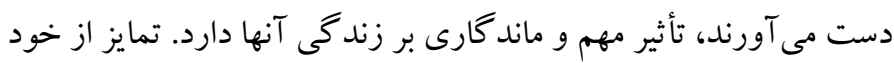

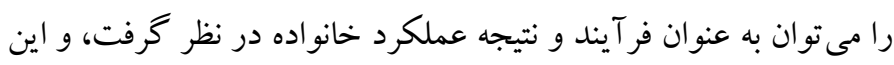

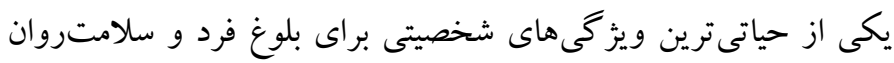
اولين بار مولتراب ارتباط ميان مفاهيمى از تئورى بين نسلى بوئن را با بيمان

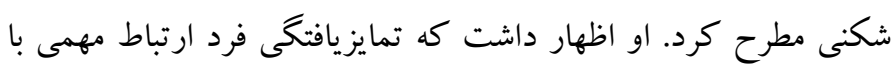

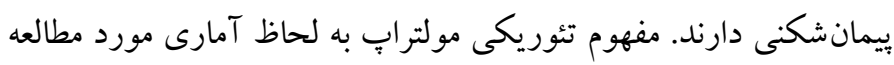

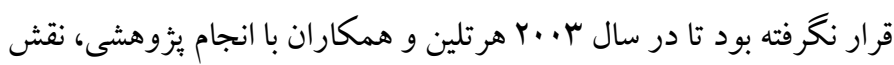
تمايزيافتحى را در بيمانشكنى تأييد كردند. همجنين فيش و و همكاردان

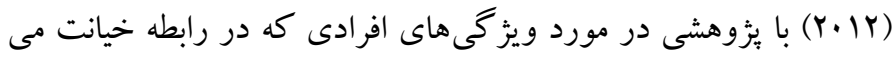

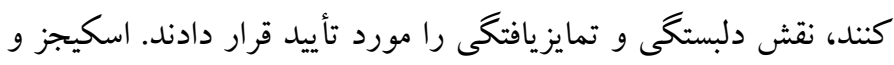

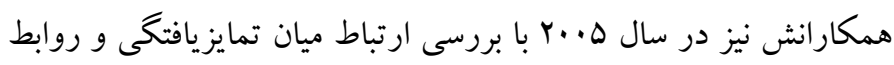

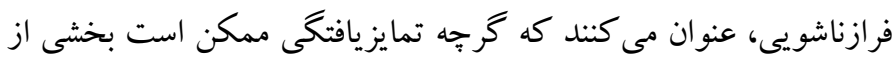

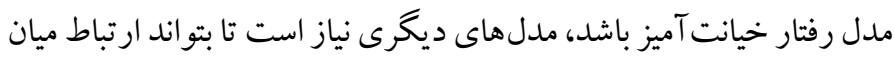

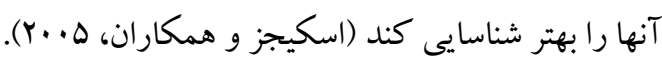

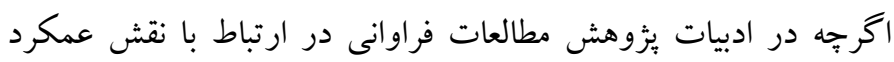

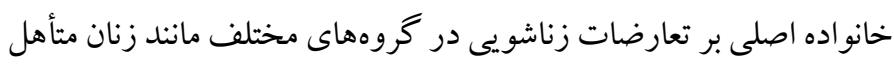
(كارخانه، فرهنگك، جزايرى، سليمانى، بهرامى و فاتحىزاده،

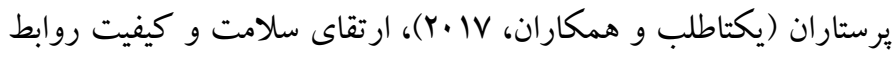
(قرمباغى و همكاران، 1ه (Y)، تعهد زناشويى' (طغرلى يور و همكاران،

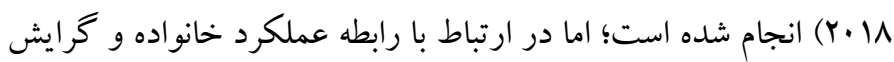
به بيمانشكنى مطالعهاى يافت نشد.

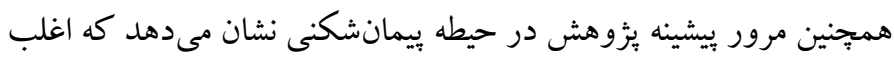

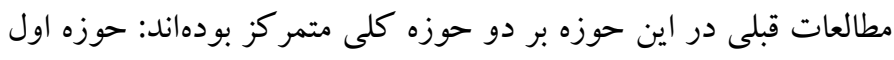

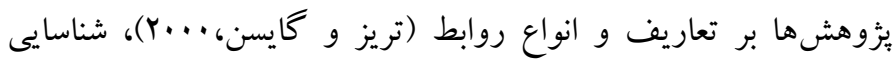

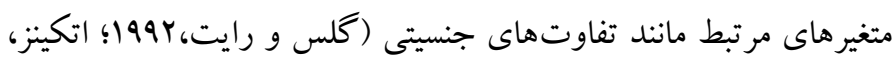

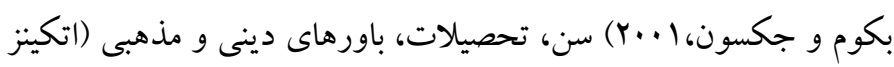

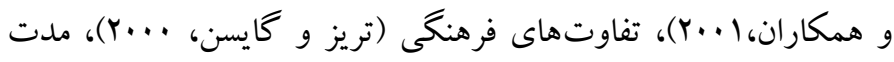
رابطه اصلى، سطح رضايتمندى جنسى و... متمر كز بوده و ارتباط آنها را

1. marital commitment 
و Y ا به صورت معكوس در نظر گرفته مى شوند. حداقل و حداكثر امتياز

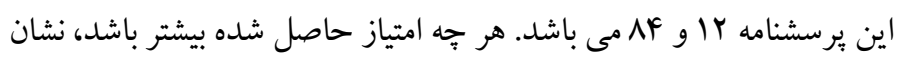

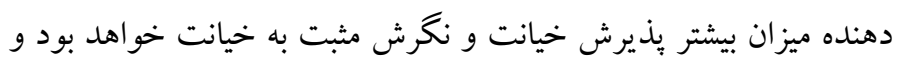

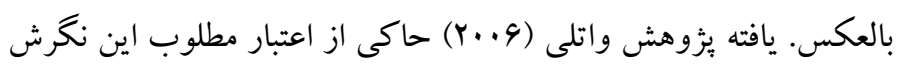

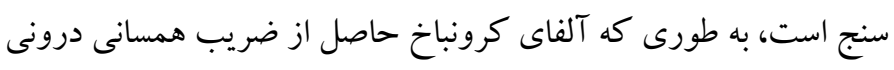

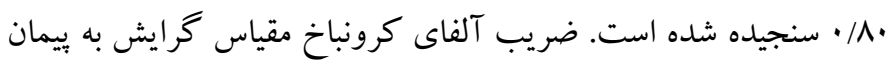

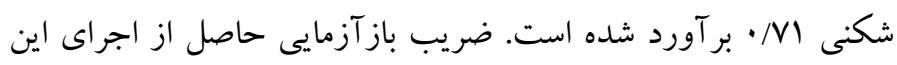

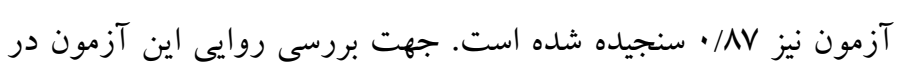

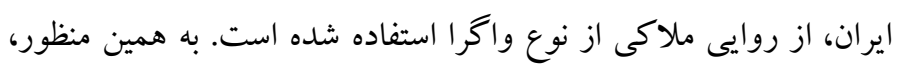

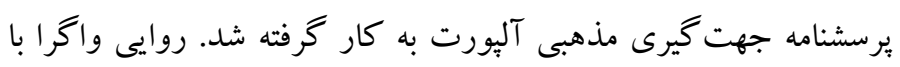

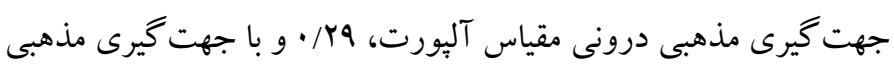

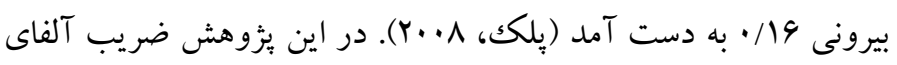

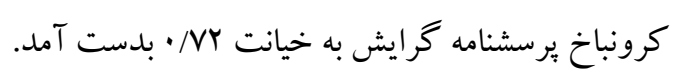

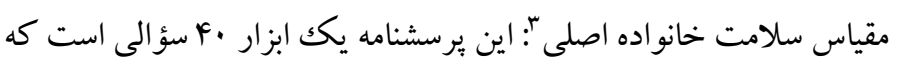

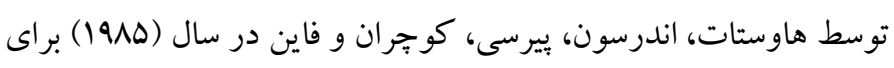

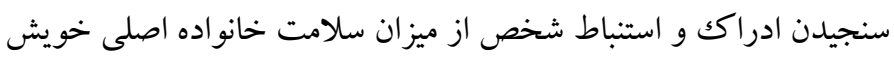

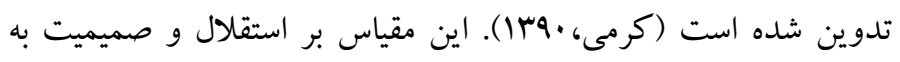

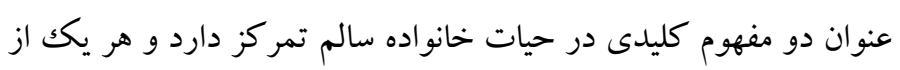

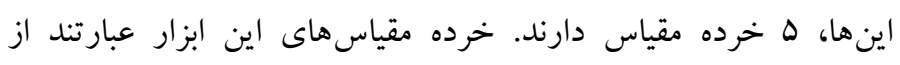

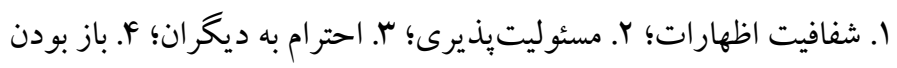

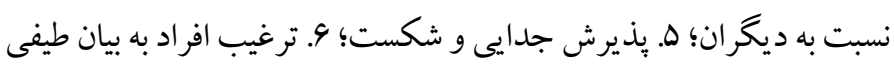

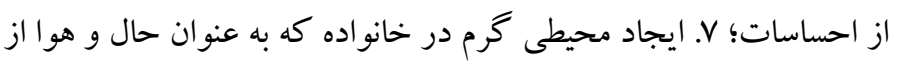
آن ياد مى شود؛ 1 حل اختلافات بدون استرس اضافى؛ 9.

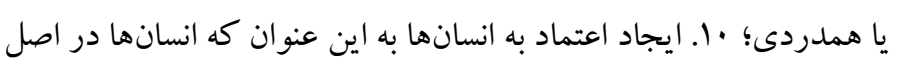

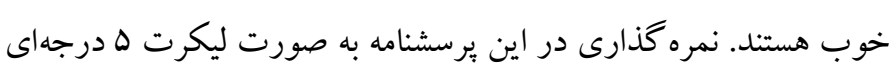

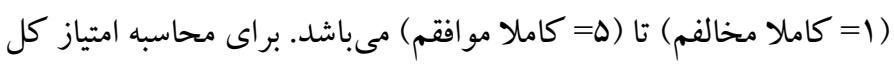

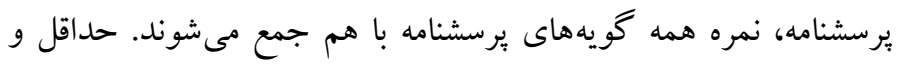

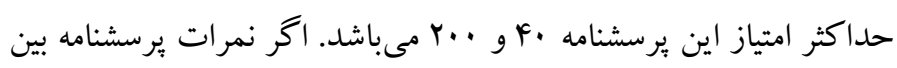

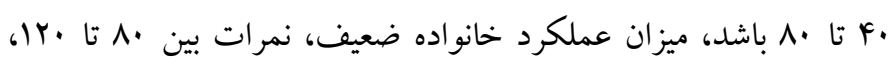

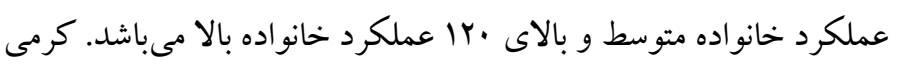

${ }^{3}$. family-of-Origin Scale
سازى معادلات ساختارى (با كاربرد نرمافزار 8.80

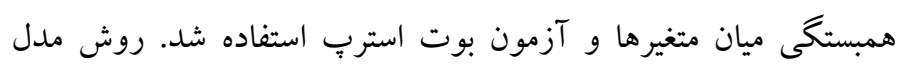

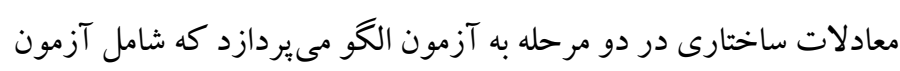

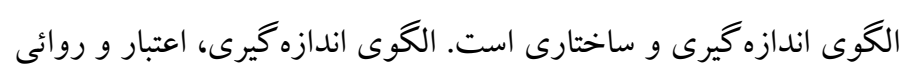

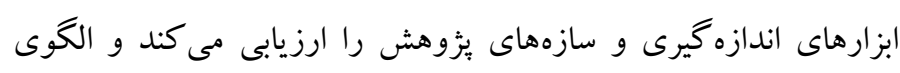
ساختارى، روابط متغير هاى مكنون را مى سنجد.

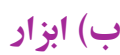
برسشنامه تمايزيافتكى خود ': برسشنامه تمايزيافتكى خود توسط اسكورن

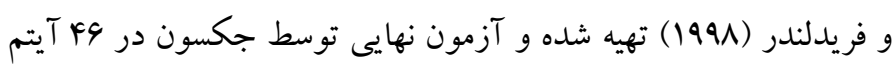
بر مبناى نظريه بوئن ساخته شده است (اسكورن و فريدلندر،

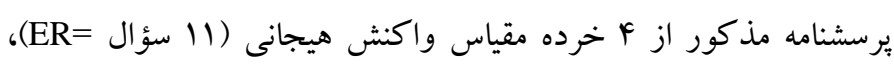

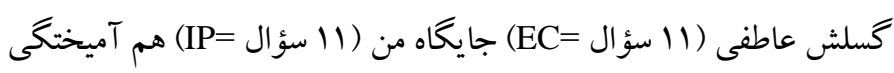

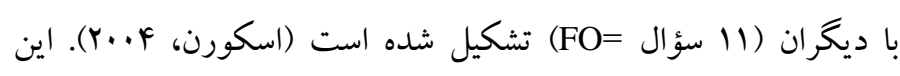

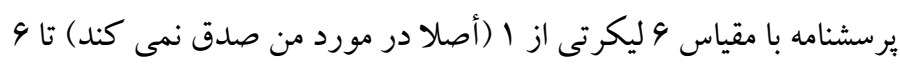
(كاملا در مورد من صدق مى كند) درجهبندى شده است و به هر سؤال

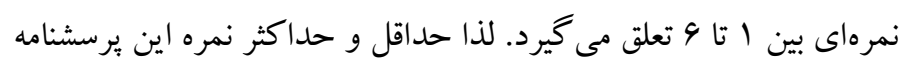

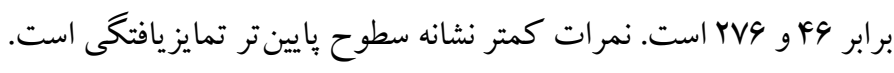

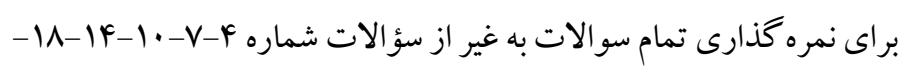

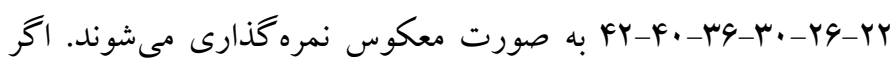

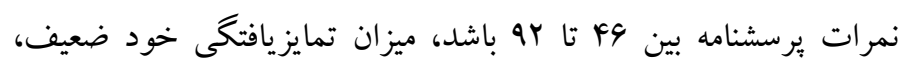

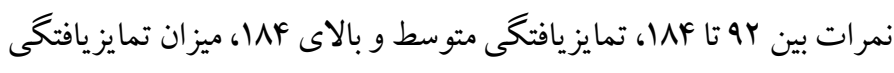

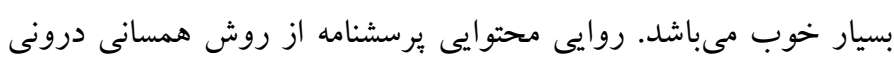

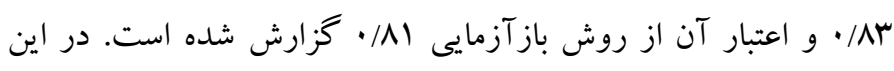

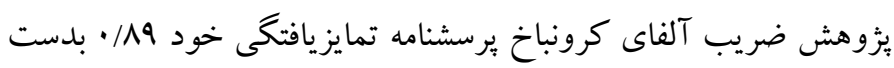
آمد.

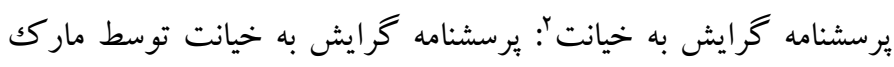

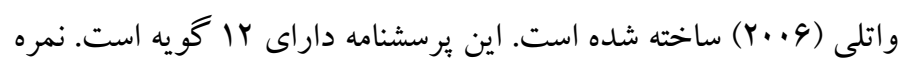

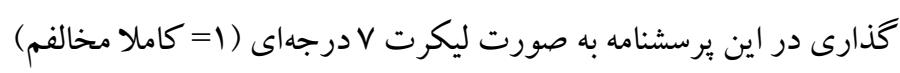

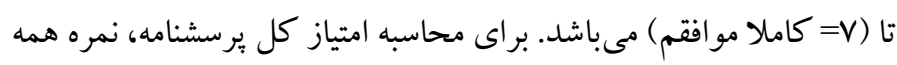

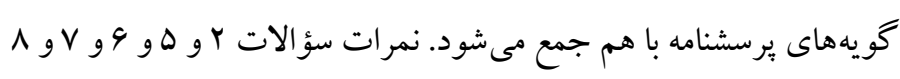

1. differentiation Of Self Inventory Revised

${ }^{2}$. attitudes toward Infidelity Scale 
باشند در تخمين صحيح مدل، مشكل ايجاد مى كنند. در خنين شرايطى بايد

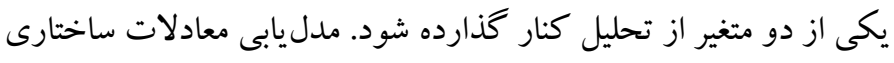

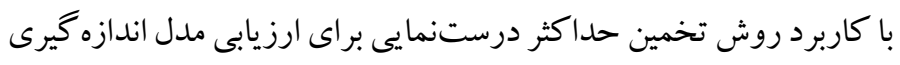
و مدل ساختاريى به كار برده شد. مدل انداز گيرى ارتباط متغيرهاى مشهود

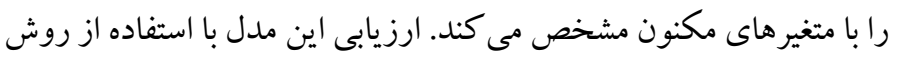
تحليل عاملى تأييدى انجام شد. شاخص هاى برازش مدل مل انداز خيرى ارئ ارائه شده در جدول ץ برازش مطلوب اين مدل را نشان مىدهد. بنابراين

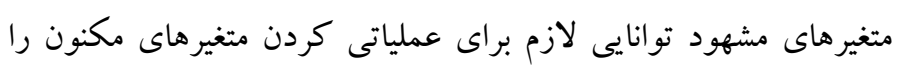
دارند. شكل ا مدل ساختارى مفهومى را به همراه ضرايب استاندارد به تصوير مى كشد. مطابق با شكل عملكرد خانو اده به ترتيب با ضريب استاندارد

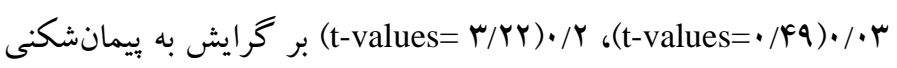

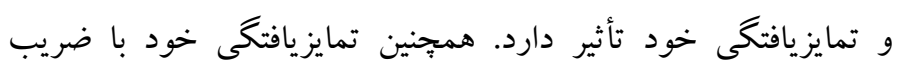

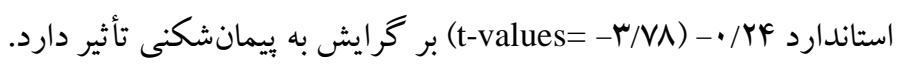
با توجه به اينكه در مدل ساختارى، معنادارى ضريب مسير با استفاده از برازي مقدار T مشخص مىشود، جنانجه مقدار t بيش از

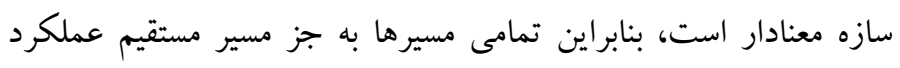
خانواده بر گرايش به ييمانشكنى معنادار است.

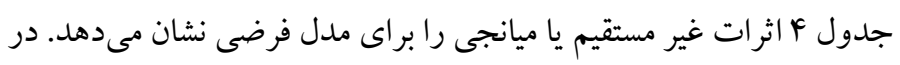
مطالعه حاضر براى ارزيابى روابط واسطهاى از آزمون بوت استرب بـ استفاده

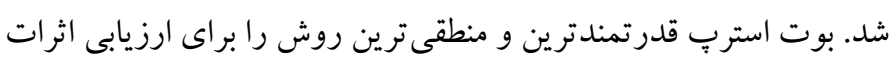

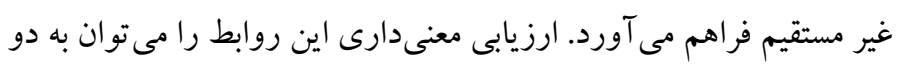

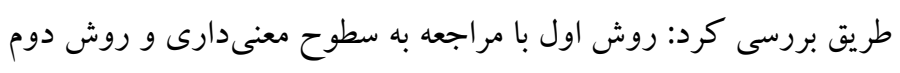
با بررسى فاصله هاى اطمينان. در صورتى كه حد بالاو يايين با فاصله اطمينان

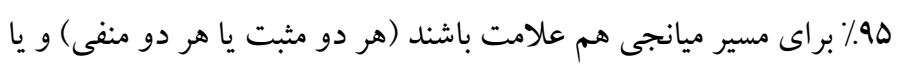

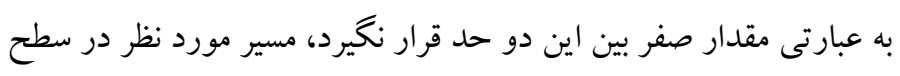
ه • • معنى دار است.

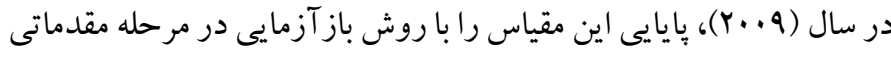

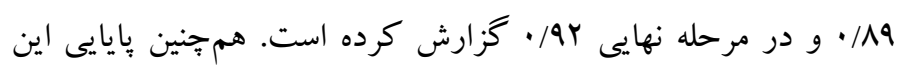

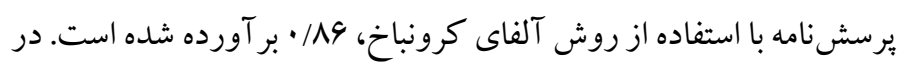

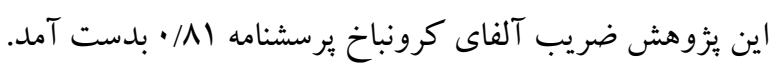

يافته ها

در اين يزوهش

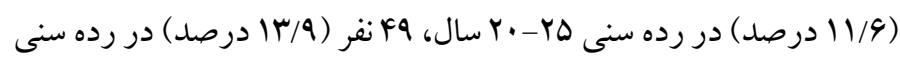

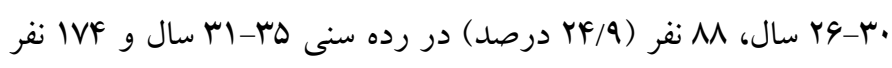

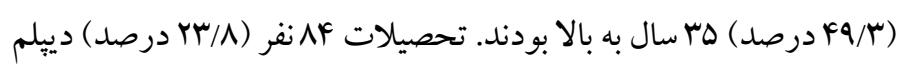

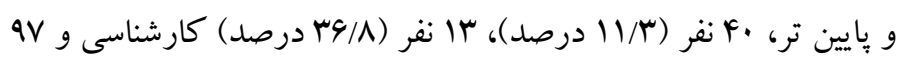

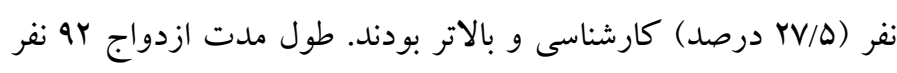

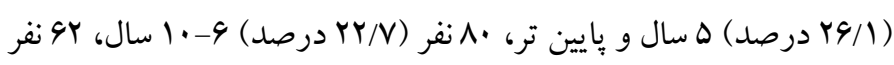
IV/9)

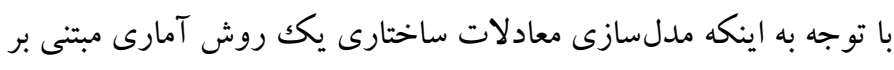

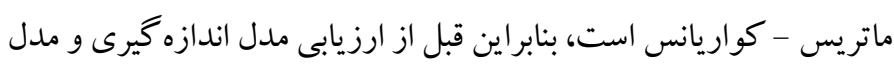
ساختارى، بيش فرضهاى مهم مدل يابى معادلات ساختارى شامل نرمال

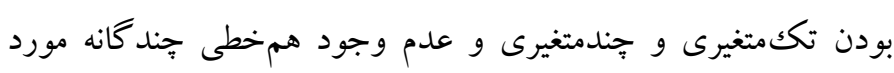

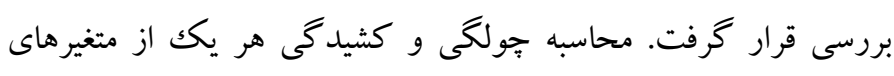

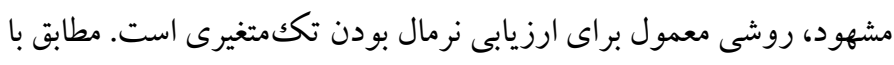

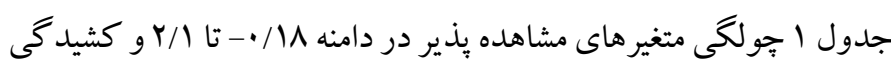

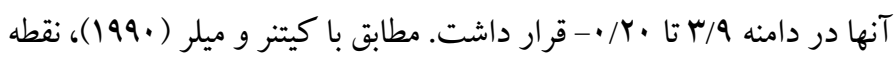

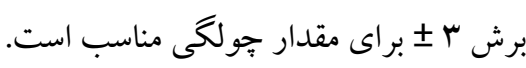

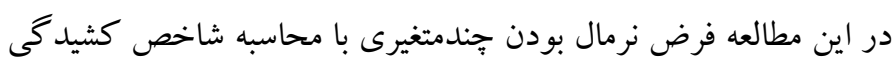

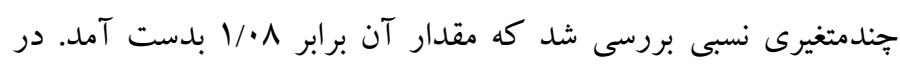

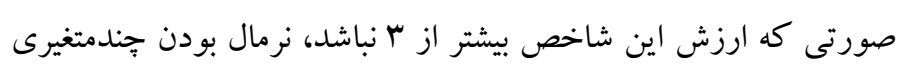

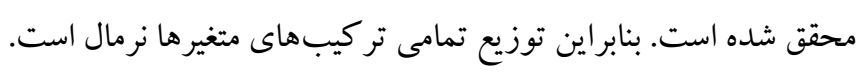

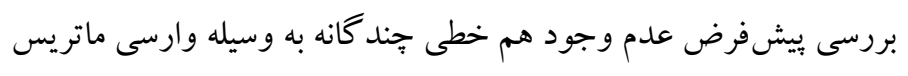

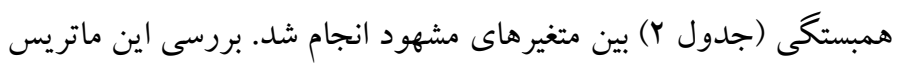

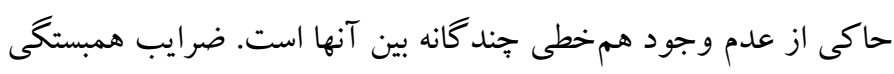

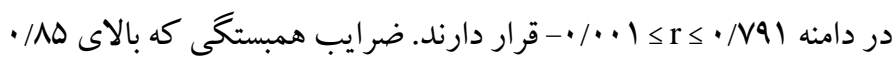




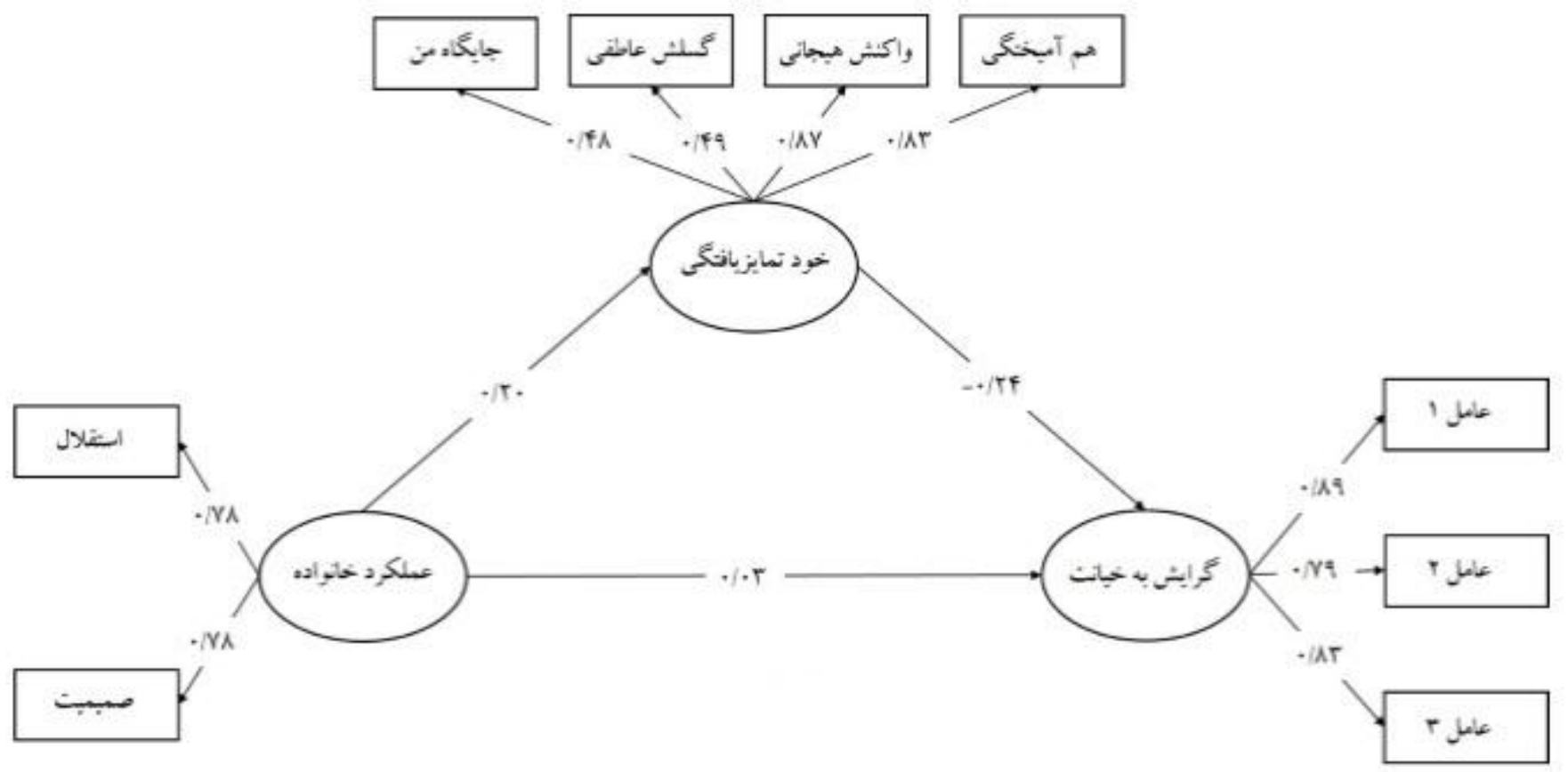

شكل 1. مدل ساختارى يزوهش

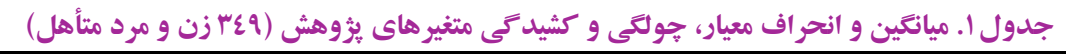

\begin{tabular}{|c|c|c|c|c|c|c|}
\hline كشيدگى & جولغى & انحرافمعيار & ميانغين & بيشينه & كمينه & متغيرها \\
\hline$-\cdot / r \cdot f$ & .11 .4 & $9 / \cdot 1$ & $M F / F A$ & 94 & ir & واكنش هيجانى \\
\hline . rar & $-\cdot / 1 \wedge 9$ & N/AF & FG/TA & 99 & IV & گَلش عاطفى \\
\hline - & .1 .99 & $\Lambda / r q$ & $r \Delta / \cdot r$ & 94 & rF & جايگاه من \\
\hline ו וr/. & . & $9 /{ }^{\prime} F$ & $r \cdot / r \mid$ & vr & 11 & آميختكى \\
\hline$r / 4 q$ & $r / \cdot r$ & $1 / 49$ & $1 / A$. & v & 1 & آيتم 1 \\
\hline$r / 99$ & $r / \cdot Y$ & $1 / 11$ & $1 / N r$ & $v$ & 1 & آيتم r \\
\hline$r / v V$ & $r / 1$. & $1 / \pi 1$ & IVY & $\checkmark$ & 1 & آيتم \\
\hline$-\cdot / F \Delta Q$ & $\cdot / T V I$ & $V / a r$ & $94 / 9 \Delta$ & $M$ & FA & استقلال \\
\hline$-\cdot / r \cdot 9$ &.$/ 1 \wedge 9$ & $1 \cdot / 0$. & $9 N / 4$. & 99 & Fi & صميميت \\
\hline
\end{tabular}

\begin{tabular}{|c|c|c|c|c|c|c|c|c|c|}
\hline 9 & $\wedge$ & $\checkmark$ & 4 & $\Delta$ & F & $r$ & r & 1 & \\
\hline & & & & & & & & 1 & 1 \\
\hline & & & & & & & 1 & ./VQI ** & r \\
\hline & & & & & & 1 & $\cdot / 194 * *$ & $\cdot / 1 \mu^{*}$ & $r$ \\
\hline & & & & & 1 &.$/ 490^{* * 4}$ & $\cdot / 1 r^{*}$ & $\cdot / \cdot \wedge \Delta$ & f \\
\hline & & & & 1 &.$/ .94$ & $\cdot / 4 \ldots * *$ & $\cdot / \cdot r$ & $\cdot / 1 \cdot 9^{*}$ & $\Delta$ \\
\hline & & & 1 & $\cdot / 4 r \Delta^{* * a}$ & $\cdot /$ r I & $\cdot / V \mid F^{* * a}$ & $\cdot / / V \cdot * *$ & ./IFY"* & 9 \\
\hline & & 1 & $-\cdot / I V Y^{* * * *}$ & $-\cdot / Y \mid \Delta^{* * *}$ &.$- / 119^{*}$ & $-\cdot / I F F^{* * * *}$ & $\cdot / \cdot r$ & 1.49 & $\checkmark$ \\
\hline & 1 & $.1991^{* *}$ & -./Irr" & $-\cdot /\left.Y Y\right|^{* \text { ** }}$ & $-\cdot / \mid F \Lambda^{* * * *}$ & $-\cdot / I I V^{*}$ & $\cdot / \cdot \wedge$ & $-\cdot / \cdot \cdot 1$ & $\wedge$ \\
\hline 1 & $\cdot / N F F * *$ & $\cdot / v \cdot \Lambda^{* * *}$ & $-\cdot / I V \Delta^{\text {*** }}$ & $-\cdot / Y V \cdot{ }^{*}{ }^{* * *}$ & $-\left.\cdot|| f\right|^{* * * x^{*}}$ & $-\cdot / I F \Lambda^{\text {典 }}$ & $-\cdot / \cdot f$ & $-. \cdot / \mu_{1}$ & 9 \\
\hline
\end{tabular}




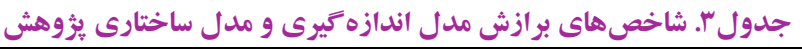

\begin{tabular}{|c|c|c|c|c|c|c|c|c|c|}
\hline AGFI & GFI & IFI & CFI & SRMR & RMSEA & $\chi^{2} / \mathrm{df}$ & df & $\mathrm{X}^{2}$ & متغير ها \\
\hline.$/ 94$ &.$/ 9 V$ &.$/ 91$ &.$/ 91$ & $.1 .9 \mathrm{~V}$ & $.1 . \Delta F$ & $Y / \cdot Y$ & r & $\Leftrightarrow 4 / \Delta \Delta$ & مدل اندازهيرى \\
\hline.$/ 94$ & $\cdot / 9 V$ & $\cdot / 91$ & $\cdot / 91$ & $.1 .9 \mathrm{~V}$ &.$/ . \Delta F$ & $Y / \cdot Y$ & r & $\forall Q / \Delta D$ & مدل ساختارى \\
\hline
\end{tabular}

جدول ع. نتايج آزمون بوت استر اب براى روابط ميانجى

\begin{tabular}{|c|c|c|c|c|c|c|}
\hline اندازه اثر & خطاى بر آورد & حد يايين & حد بالا & متغير وابسته & متغير واسط & متغير مستقل \\
\hline.$/ 4$ &.$/ \cdot r$. & $-\cdot / \cdot M$ &.$- / \cdot 1$ & ييمان شكنى & تمايزيافتگى & عملكرد خانواده اصلى \\
\hline
\end{tabular}

يكك مشكل هيجانى است، درواقع فردى كه تمايزيافتكى ضعيفى دارد با مثلثسازى، سطح اضطراب خود را كاهش داده و تلاش به حفظ رابطه

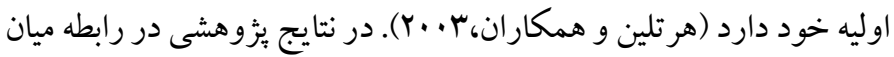
تمايز يافتكى و خشونت در ازدواج بيان مى شود كه تمايز يافتكى بالا فرد را

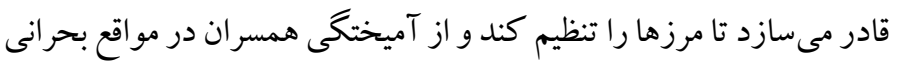

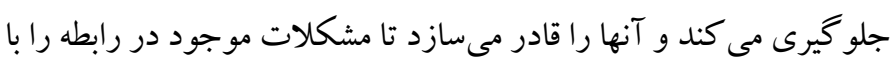
عقل و منطق و نه براساس واكنش يذيرى هاى هيجانى و احساسى رفع كنند. در سمت مقابل، زوجهاى تمايزنيافته با واكنشيذيرى هيجانى و فاصله مائه

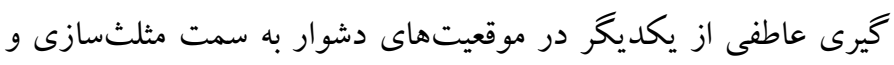

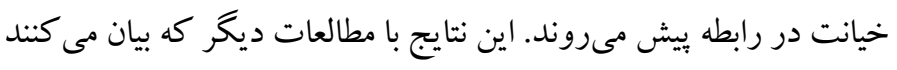

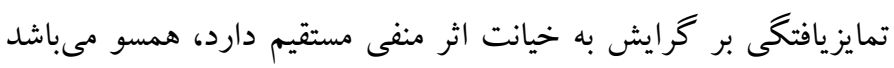
(زارعى و همكاران، سجبا؛ كلاد، 1999).

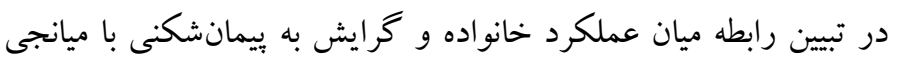
كرى تمايزيافتخى عنوان مى شود كه خانو اده به عنوان نخستين بايگاه شكل

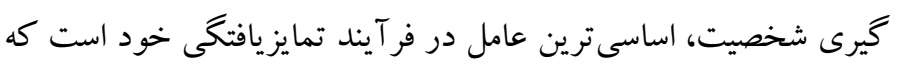

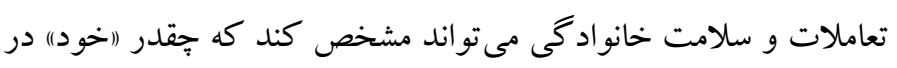

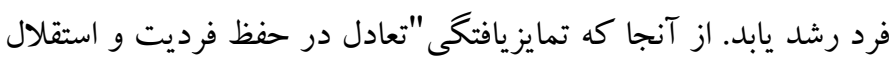

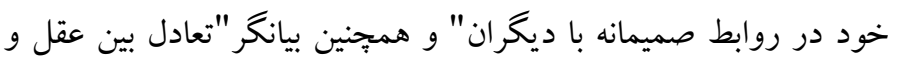
هيجان" است، خانوادههايى كه از سلامت خوبى برخوردار هستند، ميزان

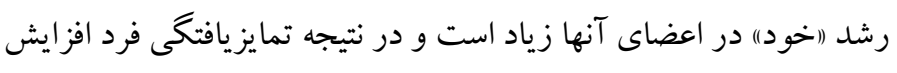
مى يابد و مستقل تر عمل مى كند. افراد تمايزيافته تعريف مشخصى از خود

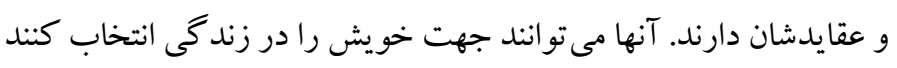

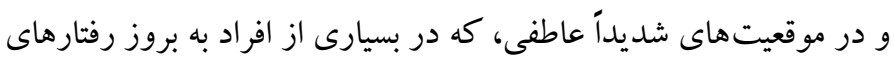

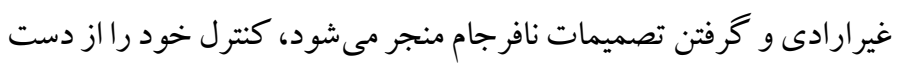

بحث و نتيجه كيرى

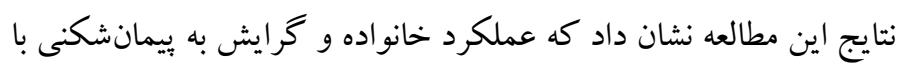

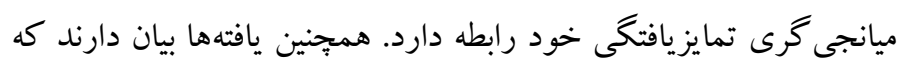

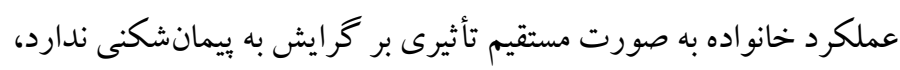

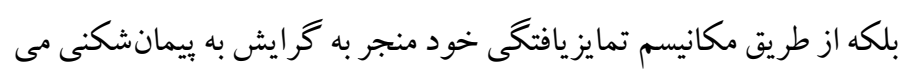

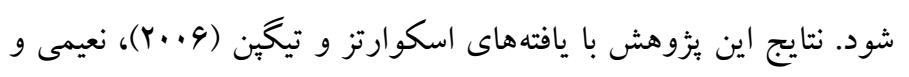

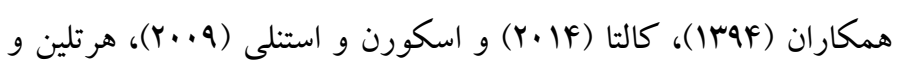

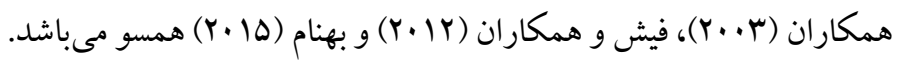

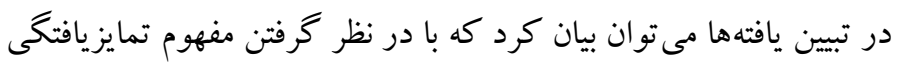

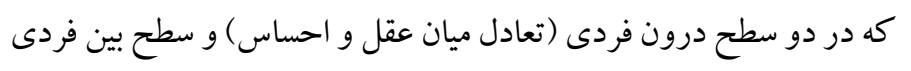

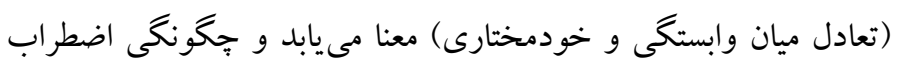

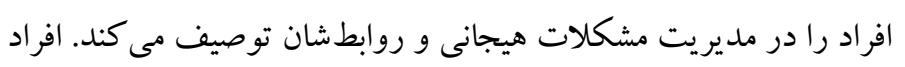

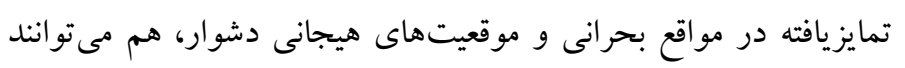

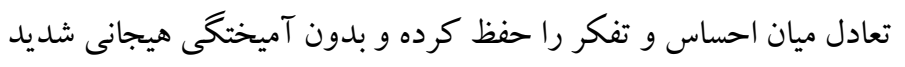

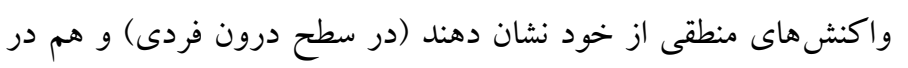

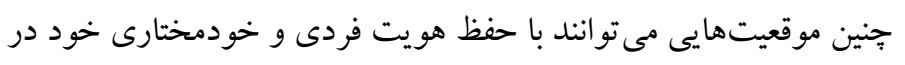

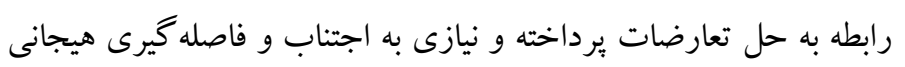

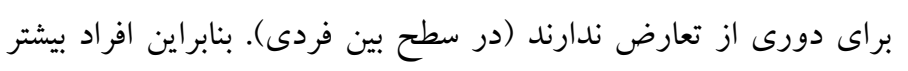

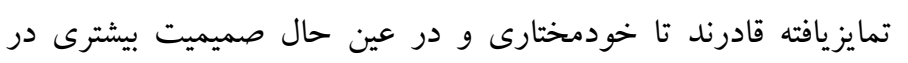
روابطشان بدون غرق شدن در احساسات داشته باشند (جانغك و كايل،

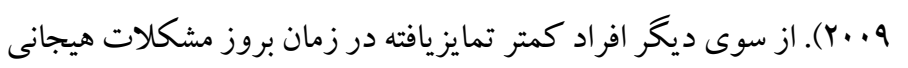
در روابطشان، با برقرارى ارتباط با فردى غير از همسر با ايجاد فاصله هيجانى با همسر خود، سعى در حفظ تعادل دايناميك خود در رو رابطه دارند.

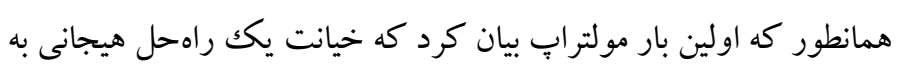


با اين كه يافتها از مدل فرضى بزوهش حمايت كردند، نتايج اين بزوهش

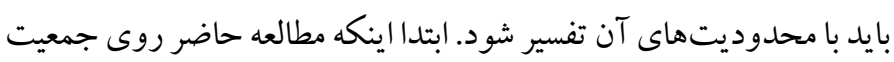

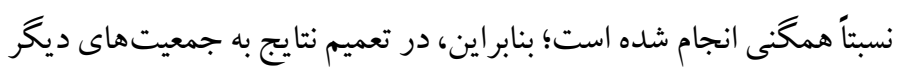

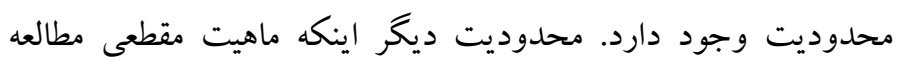

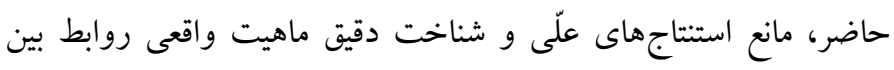

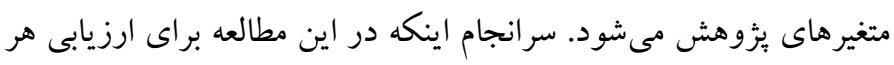

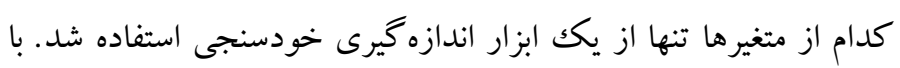

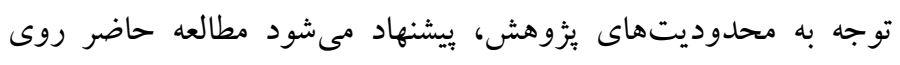

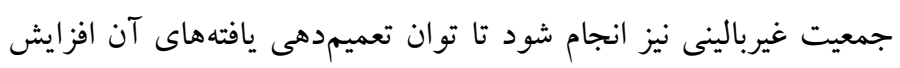

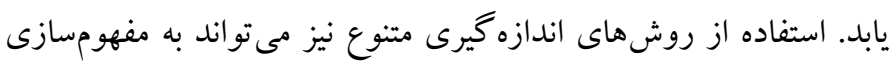
بهتر متغير كمك كند. همجنين به منظور استنتاج روابط على و شفاف كردن تو الى زمانى بين متغيرهاى يزولهش، انجام مطالعات طولى ييشنهاد مى شود.

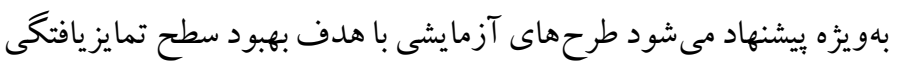

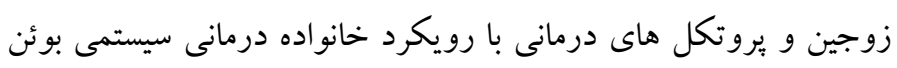

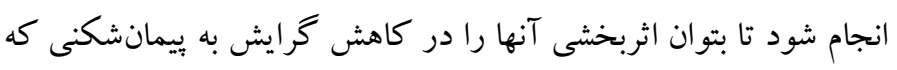

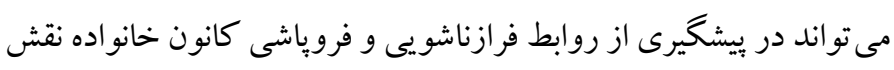

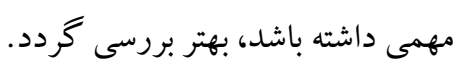

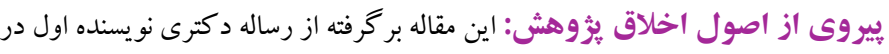

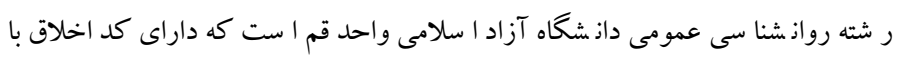
شـاره IR.IAU.QOM.REC.1399.058 است و مشار كت كند كان آكاهاهانه و داوطلبانه

در يُزوهش مشار كت نمودند.

حامى مالى: اين يزوهش در قالب رساله دكترى بدون حمايت مالى است.

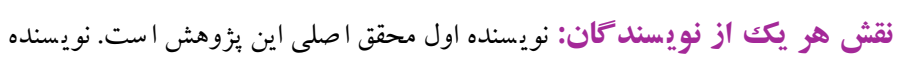

$$
\text { دوم استاد راهنما و نويسنده سوم استاد مشاور است. }
$$

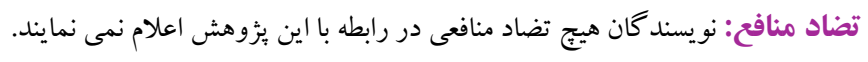

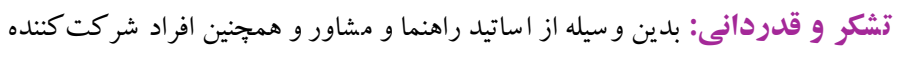
در اين تحقيق تقدير و تشكر مى كنم.
ندهند و با در نظر گرفتن عقل و منطق تصميم گيرى كنند. در مقابل، افراد

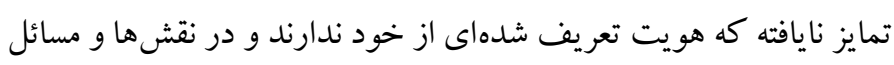
بين شخصى موجود همراه با موج عاطفى خانواده حركت مى كنند،

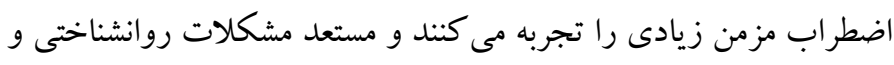

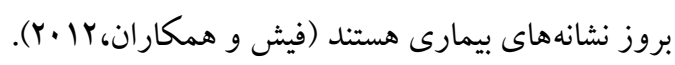

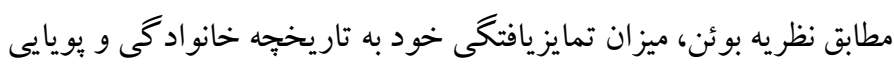

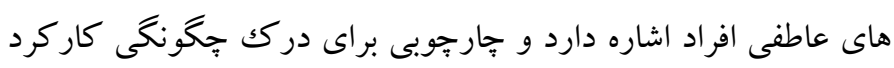

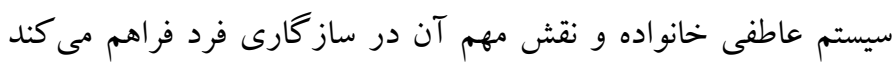

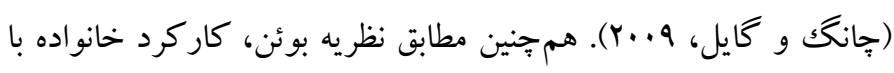

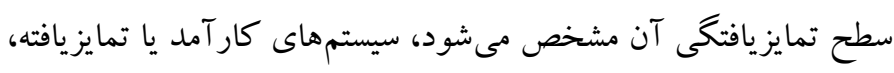

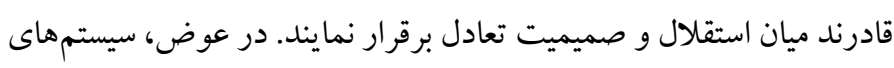
تمايزنايافته با آميختكى هيجانى و شكست در ايجاد تعادل كار آمد ميان نياز

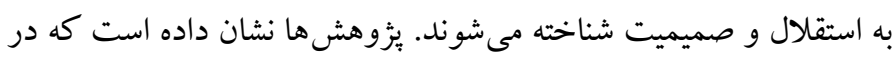

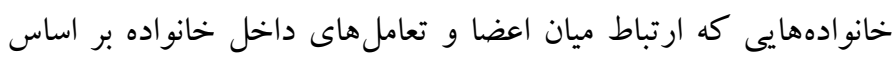
نزديكى و صميميت و تفاهم بين افراد استوار است، همه اعضا نسبتاً عليه

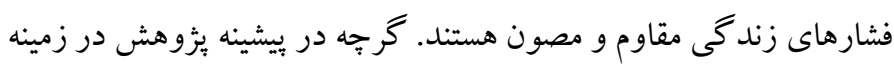

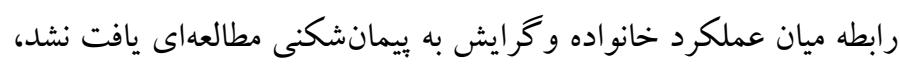

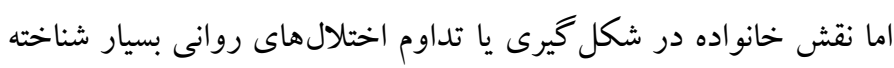

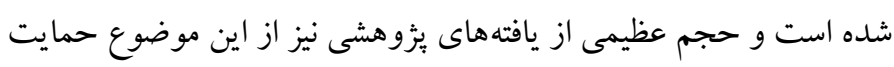

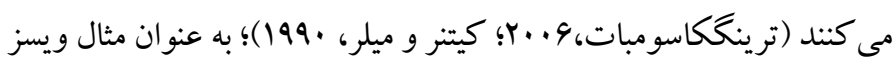

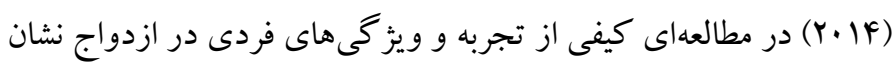

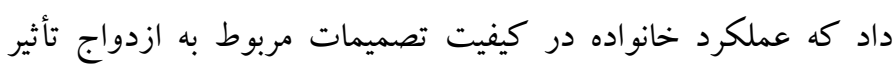

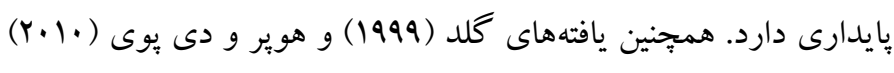

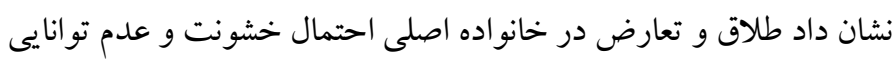
حل مسأله، عدم اعتماد و از هم گسيختخى زند ييشبينى مى كند. در واقع تجارب افراد در خانواده اصلى نشاندهنده اين

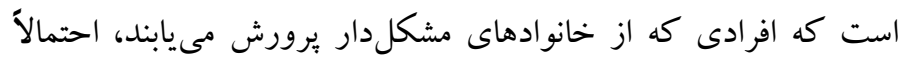
مستعد تكرار تجارب و مشاهدات گذشته در زندگى مشترك خود هود هستند. 


\section{References}

Atkins, D. C., Baucom, D. H., \& Jacobson, N. S. (2001). Understanding infidelity: Correlates in a national random sample. Journal of family psychology, 15(4), 735. [Link]

Behnam, N. (2015). The relationship between differentiation and infidelity among IranianAmericans (Dissertation for Doctor of Philosophy) Alliant International University Los Angeles. [Link]

Blow, A. J., \& Hartnett, K. (2005). Infidelity in committed relationships ii: A substantive review. Journal of marital and family therapy, 31(2), 217-233. [Link]

Buser, T. J., Pertuit, T. L., \& Muller, D. L. (2019). Nonsuicidal self-injury, stress, and selfdifferentiation. Adultspan Journal, 18(1),4-16. [Link]

Chung, H., \& Gale, J. (2009). Family functioning and selfdifferentiation:A cross-cultural examination. Contemporary Family Therapy, 31(1), 19-33. [Link]

Fish, J. N., Pavkov, T. W., Wetchler, J. L., \& Bercik, J. (2012). Characteristics of those who participate in infidelity: The role of adult attachment and differentiation in extradyadic experiences. The American Journal of Family Therapy, 40(3), 214229. [Link]

Gharehbaghy, F. (2011). An investigation into Bowen family systems theory in an Iranian sample. Iranian Journal of Psychiatry and Behavioral Sciences, 5(1), 56-63. [Link]

Gharehbaghy, F., Besharat, M,. Rostami, R., \& Gholamali Lavasani M. (2015). Designing and evaluating an intervention based on Bowen family systems theory to improve health and quality of relationships. Journal of Psychological Sciences, 14 (54), p. 168179. (Persian). [Link]

Glad, G. E. (1999). The effect of self-differentiation on marital conflict. American journal of pastoral counseling, 2(4), 65-76. [Link]

Glass, S. P., \& Wright, T. L. (1992). Justifications for extramarital relationships: The association between attitudes, behaviors, and gender. Journal of sex Research, 29(3), 361-387. [Link]

Hertlein, K. M., Ray, R., Wetchler, J. L., \& Killmer, J. M. (2003). The role of differentiation in extradyadic relationships. Journal of Couple \& Relationship Therapy, 2(4), 33-50. [Link]

Hertlein, K. M., \& Skaggs, G. (2005). Assessing the relationship between differentiation and infidelity:
A structural equation model. Journal of Couple \& Relationship Therapy, 4(2-3), 195-213. [Link]

Hooper, L. M., \& DePuy, V. (2010). Mediating and moderating effects of differentiation of self on depression symptomatology in a rural community sample. The Family Journal, 18(4), 358-368. [Link]

Imanizad, A., Golmohammadian, M., Moradi, O., \& Goodarzi, M. (2021). The effectiveness of emotionoriented couple therapy on forgiveness and communication beliefs of couples involved in marital infidelity. Journal of Psychological Sciences, 20 (100), 653-665. (Persian). [Link]

Kaleta, K. (2014). Marital satisfaction, differentiation of self and stress perceived by women. In Polskie Forum Psychologiczne, 19(3), 305-319. [Link]

Karami, A. (2011). Psychometric charactristic and standardization of health level of family-of-original scale. Consulting Research and Development, 38. 103-118. (Persian). [Link]

Karkhaneh, P., Farhang, M., Jazayeri, R., Soleimani, R., Bahrami, F., \& Fatehizade, M. (2016). The Study of the effectiveness of the Bowen self-differentiation counseling on marital conflicts on married women in Isfahan. International Journal of life science \& pharma research. 6(4), 13-24. [Link]

Keitner, G. I., \& Miller, I. W. (1990). Family functioning and major depression: an overview. The American Journal of Psychiatry. [Link]

Lampis, J., Cataudella, S., Agus, M., Busonera, A., \& Skowron, E. A. (2019). Differentiation of self and dyadic adjustment in couple relationships: A dyadic analysis using the actor-partner interdependence model. Family process, 58(3), 698-715. [Link]

Lee, H. S., \& Ok, S. W. (2002). Family of Origin Influences on Anxiety, Open Communication, and Relationship Satisfaction-A Test of Bowenian theory of Anxiety as a Mediator in the Intergenerational Transmission. International Journal of Human Ecology, 3(1), 111-126. [Link]

Muraru, A. A., \& Turliuc, M. N. (2012). Family-of-origin, romantic attachment, and marital adjustment: a path analysis model. Procedia-Social and Behavioral Sciences, 33, 90-94. [Link]

Naimi, Q., Pirsaghi, F., \& Arabzadeh, M. (2016). Investigating the structural relationship between main family health, emotional schema, emotion regulation and desire to marry in students with the role of mediating their differentiation. Quarterly of Cultural Education of Women and Family, 10 (33), 43-64. (Persian). [Link] 
Peleg, O. (2008). The relation between differentiation of self and marital satisfaction: What can be learned from married people over the course of life?. The American journal of family therapy, 36(5), 388-401. [Link]

Priest, J. B. (2019). Examining differentiation of self as a mediator in the Biobehavioral Family Model. Journal of marital and family therapy, 45(1), 161175. [Link]

Schwartz, J. P., Thigpen, S. E., \& Montgomery, J. K. (2006). Examination of parenting styles of processing emotions and differentiation of self. The Family Journal, 14(1), 41-48. [Link]

Skowron, E. A., \& Dendy, A. K. (2004). Differentiation of self and attachment in adulthood: Relational correlates of effortful control. Contemporary family therapy, 26(3), 337-357. [Link]

Skowron, E. A., \& Friedlander, M. L. (1998). The Differentiation of Self Inventory: Development and initial validation. Journal of counseling psychology, 45(3), 235. [Link]

Skowron, E. A., Stanley, K. L., \& Shapiro, M. D. (2009). A longitudinal perspective on differentiation of self, interpersonal and psychological well-being in young adulthood. Contemporary Family Therapy, 31(1), 3-18. [Link]

Toghroli Pour Grighani, M., Mousavi Nasab, S. M., \& Rahmati, A. (2018). Examination of a Causal Model of Family-of-Origin's Health, Attachment Styles, and Marital Commitment with the Mediating Role of Self-Differentiation. International Journal of Behavioral Sciences, 12(3), 91-95. [Link]

Trangkasombat, U. (2006). Family functioning in the families of psychiatric patients: a comparison with nonclinical families. Journal-Medical Association of Thailand, 89(11), 1946. [Link]

Treas, J., \& Giesen, D. (2000). Sexual infidelity among married and cohabiting Americans. Journal of marriage and family, 62(1), 48-60. [Link]

Weiss, J. M. (2014). Marital Preparation, Experiences, and Personal Qualities in a Qualitative Study of Individuals in Great Marriages. (master's thesis). Utah State University. USA. [Link]

Whatley, M. (2006), Attitudes toward Infidelity Scale, Department of Psychology, Valdosta State University. [Link]

Yektatalab, S., Seddigh Oskouee, F., \& Sodani, M. (2017). Efficacy of Bowen theory on marital conflict in the family nursing practice: A randomized controlled trial. Issues in mental health nursing, 38(3), 253260. [Link]
Zarei, S \& Hosseingholi, F (2014). The Prediction of Marital Commitment based on Self- Conscious Affects (Shame and Guilt) and Self-Differentiation among Married University Students. Journal of Family Counseling and Psychotherapy, 4 (1), 133114. (Persian). [Link] 